\title{
Tecnologias para o tratamento de dejetos de suínos
}

\author{
Paulo Belli Filho1 , Armando B. de Castilhos Jr. ${ }^{2}$, Rejane H.R. da Costa ${ }^{3}$, Sebastião R. Soares ${ }^{4}$ \& Carlos C. Perdomo ${ }^{5}$ \\ 1 Departamento de Engenharia Sanitária e Ambiental da UFSC. Campus Universitário - Bairro Trindade, CEP 88010-970, \\ Florianópolis, SC. Fone: (48) 331-9597. E-mail: belli@ens.ufsc.br (Foto) \\ 2 Departamento de Engenharia Sanitária e Ambiental da UFSC. E-mail: borges@ens.ufsc.br \\ ${ }^{3}$ Departamento de Engenharia Sanitária e Ambiental da UFSC. E-mail: rejane@ens.ufsc.br \\ ${ }^{4}$ Departamento de Engenharia Sanitária e Ambiental da UFSC. E-mail: soares@ens.ufsc.br \\ ${ }^{5}$ CNPSA/EMBRAPA. CP 21, CEP 89700-000, Concórdia, SC. Fone: (49) 442-8555. E-mail: perdomo@cnpsa.embrapa.br
}

Protocolo $074-29 / 06 / 2000$

\begin{abstract}
Resumo: Com este trabalho apresentaram-se os problemas ambientais provenientes da suinocultura no Sul do Brasil, as formas adotadas de criação dos animais e suas relações econômicas, sociais e ambientais. No Estado de Santa Catarina concentra-se o maior rebanho nacional, da ordem de 4,5 milhões de cabeças, gerando aproximadamente 150.000 empregos diretos, possuindo 25.000 propriedades produtoras de animais. Em termos ambientais, a suinocultura é um setor com baixa qualidade ambiental, pois apenas $15 \%$ das propriedades dispõem, de alguma forma, de manejo de dejetos. Com o objetivo de contribuir na busca da qualidade ambiental para este setor, apresentam-se orientações tecnológicas, definidas a partir de resultados de pesquisas, com metodologias para reduzir a poluição ambiental em função das caraterísticas da propriedade, das localidades e da tendência mundial.
\end{abstract}

Palavras-chave: tratamento de dejetos, poluição pela suinocultura, suinocultura

\section{Technology for swine waste treatment}

\begin{abstract}
In this work the environmental problems related with hog raising in southern Brazil, the adopted ways of animal care and management, as well as their economic, social and environmental relations are discussed. The highest concentration of national livestock is in the State of Santa Catarina (about 4.5 million), generating approximately 150,000 direct jobs in its 25,000 rural properties. As far as the environment is concerned, hog raising is a sector of low environmental quality, and it covers a wider regional area when compared to other economic activities. Only $15 \%$ of the property holders perform some sort of waste management, and in many cases it is carried out in an inappropriate way. Both guidelines and study results are shown in this work, in search of a higher environmental quality for this sector and in order to prevent and to control environmental pollution according to the farms' characteristics, their locations and the global practices.
\end{abstract}

Key words: waste treatment, pork production, pig pollution

\section{INTRODUÇÃO}

O Brasil possui o quarto plantel de suínos do mundo, com um efetivo de 34 milhões de cabeças. Por outro lado, o Estado de Santa Catarina, com aproximadamente 4,5 milhões, é o maior produtor regional da América Latina. Por conseqüência, a suinocultura é um setor que contribui, de maneira significativa, com a economia estadual, apresentando importância social, econômica e cultural muito grande em regiões com tradição nesta atividade, porém é um setor com baixa qualidade ambiental, poluindo as águas, os solos, afetando a qualidade do ar nestas regiões, através da emissão de maus odores, e pela proliferação descontrolada de insetos, ocasionando muito desconforto ambiental às populações.
A produção no Estado é distribuída em aproximadamente 21.000 pequenas propriedades, 3.500 médias e 500 grandes, gerando $10^{7} \mathrm{~m}^{3}$ de dejetos ao ano. Somente um pequeno percentual deste produto possui manejo adequado que respeita as condições de valorização, sem poluir o meio ambiente. Neste trabalho são apresentados problemas relacionados aos sistemas de produção de suínos em Santa Catarina e as principais técnicas de manejo dos dejetos desenvolvidas através de pesquisas, com o objetivo de contribuir para a sustentabilidade da suinocultura catarinense. As pesquisas se incluem como atividades de um programa de gestão ambiental dos dejetos de suínos, elaborado entre o Centro Nacional de Pesquisas em Suínos e Aves da Empresa Brasileira de Pesquisa Agropecuária 
(CNPSA/EMBRAPA) e o Departamento de Engenharia Sanitária e Ambiental da Universidade Federal de Santa Catarina.

\section{SISTEMAS DE PRODUÇÃO DE SUÍNOS E O MEIO AMBIENTE}

Em Santa Catarina, a produção de suínos é caracterizada por um nível intenso de confinamento dos animais e, como conseqüência, produz elevada quantidade de dejetos. Outro aspecto é a capacidade-suporte do solo, na medida em que este é utilizado sem plano de aplicações e destinado para fins de produção agrícola não integrada como alternativa de seu tratamento e controle da poluição. Avaliações preliminares indicam que apenas $15 \%$ dessas propriedades possuem metodologias de manejo através da valorização e tratamento dos dejetos; o restante é destinado ao meio natural, degradando os recursos hídricos. Parte das tecnologias empregadas para o tratamento dos dejetos não é projetada, construída nem operada de maneira adequada.

Os parâmetros de projetos empregados são copiados da literatura estrangeira ou adaptados de outros resíduos para a suinocultura. Outros fatores contribuem para este quadro, tais como: falta de formação de pessoal, de orientação técnica dos produtores e ausência de controle ambiental pelos órgãos responsáveis, apesar da disponibilidade de legislação avançada. Neste contexto, as principais conseqüências são: a degradação ambiental pela contaminação das águas superficiais e subterrâneas, a poluição orgânica pelo nitrogênio, a presença de microrganismos enteropatogênicos, a alteração das características dos solos e a poluição do ar, pela emissão de maus odores, e a presença de insetos.

\section{TECNOLOGIAS AVALIADAS PARA O CONTROLE DA POLUIÇÃO}

A problemática descrita é resultado da inexistência de um programa de sustentabilidade da suinocultura no Brasil, que integre os produtores de suínos e as agroindústrias. A resolução desses problemas passa pelo estabelecimento de estratégias que integrem os componentes: humano (formação de recursos humanos), técnico (desenvolvimento de metodologias e tecnologias) e de sensibilização (educação ambiental). Atualmente, existem esforços de desenvolvimento de pesquisas, direcionados à valorização e ao tratamento dos dejetos de suínos, cujos principais resultados são apresentados abaixo.

\section{Produção de suínos sobre camas biológicas para animais confinados}

Esta metodologia de criação de suínos apresenta, como principal vantagem, a redução dos volumes dos dejetos produzidos e, por conseqüência a redução da poluição ambiental. O sistema de criação de suínos sobre camas consiste no armazenamento dos dejetos na camada do substrato, o qual absorve por completo esses produtos. A camada possui altura variável (em média $0,50 \mathrm{~m}$ ) mantida através da reposição de material durante a permanência dos animais. Segundo Simoni (1992) é possível a manutenção da cama na criação de suínos por um período de até 18 meses. Este sistema é mais empregado na fase de crescimento e terminação dos suínos (animais com peso inicial entre $18-25 \mathrm{~kg}$, permanecendo na cama até os $105 \mathrm{~kg}$ ).

Por outro lado, Huysman et al. (1992) afirmam que o tempo de permanência depende não somente das condições climáticas de cada país e do manejo do sistema como também, do regime de alimentação dos animais. Durante este período não ocorre uma completa estabilização da matéria orgânica, uma vez que o substrato recebe reposição de material de composição da cama para manter a altura inicial e, igualmente, os dejetos, ao longo do tempo de permanência dos animais. A Figura 1 apresenta um esquema do sistema de camas, segundo modelo implementado no Estado de Santa Catarina, onde se verifica o movimento realizado sobre os animais, para revolvimento do substrato.

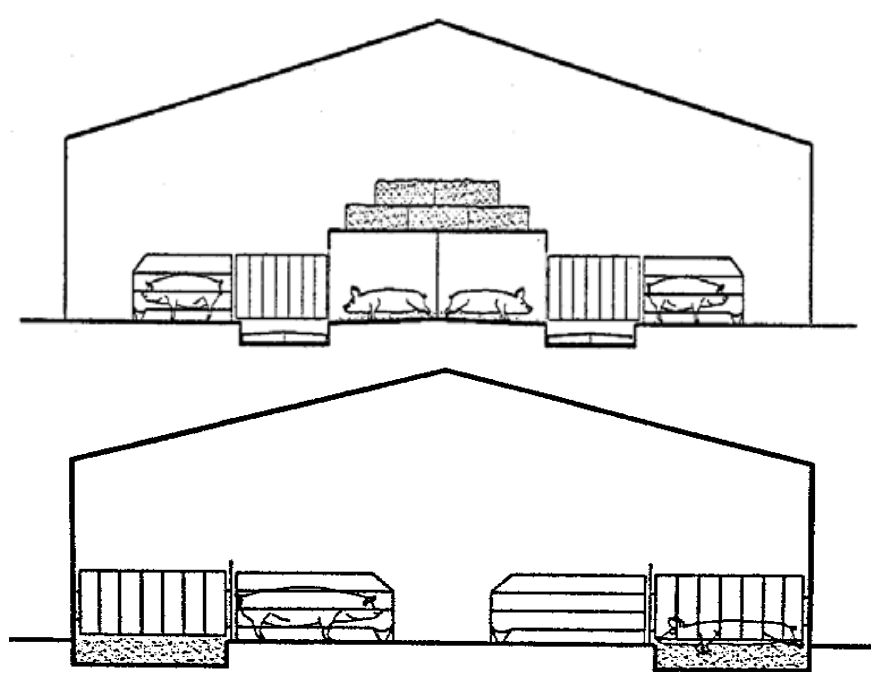

Figura 1. Esquema de instalações para criação de suínos com camas biológicas

Os trabalhos de pesquisa, desenvolvidos inicialmente, foram direcionados para avaliar os materiais a serem utilizados como substrato nas camas biológicas (serragem de madeira, sabugo de milho, palha de arroz e maravalha). Tumelero (1998) apresentou os resultados de estudos que mostraram a possibilidade de utilização desses materiais como substrato das camas biológicas para os animais e valorização dos produtos finais na agricultura. Todos os materiais apresentam bom desempenho, no que se refere à absorção, com exceção da casca de arroz. A Tabela 1 apresenta as características dos materiais analisados, após 9 meses de estudo.

Esta metodologia de produção de suínos poderá confirmar-se como excelente solução para o tratamento dos dejetos de suínos. Neste caminho de pesquisas, Goulart (1997) demonstrou também a eficiência do processo de compostagem dos materiais que compõem as camas biológicas e, igualmente, o comportamento dos animais neste método de produção animal. Os dados obtidos em ensaios realizados na Estação Experimental do CNPSA/EMBRAPA, mostram que o sistema de camas biológicas se mostra eficiente quanto à diminuição da quantidade de dejetos sólidos e líquidos, totalmente absorvidos pelo leito.

Apesar dessas características, verificou-se que a degradação do substrato que compõe o sistema de camas durante a fase de criação de suínos não é suficiente para 
Tabela 1. Parâmetros iniciais e finais dos diferentes materiais utilizados nas camas biológicas (Tumulero, 1998)

\begin{tabular}{|c|c|c|c|c|}
\hline \multirow[b]{2}{*}{ Parâmetros ${ }^{*}$} & \multicolumn{4}{|c|}{ Material } \\
\hline & $\begin{array}{c}\text { Casca de } \\
\text { Arroz }\end{array}$ & Maravalha & $\begin{array}{l}\text { Sabugo } \\
\text { de Milho }\end{array}$ & Serragem \\
\hline Umidade $_{\mathrm{i}}(\%)$ & 9,97 & 10,51 & 7,30 & 54,80 \\
\hline Umidade $_{\mathrm{f}}(\%)$ & 40,61 & 47,99 & 38,38 & 49,83 \\
\hline Carbono $_{i}(\%)$ & 39,37 & 46,17 & 47,31 & 23,35 \\
\hline Carbono $_{\mathrm{f}}(\%)$ & 16,84 & 17,85 & 22,34 & 17,93 \\
\hline Nitrogênio $_{i}(\%)$ & 0,46 & 0,09 & 0,54 & 0,13 \\
\hline Nitrogênio $_{\mathrm{f}}(\%)$ & 1,02 & 0,93 & 1,84 & 0,80 \\
\hline$(\mathrm{M} \mathrm{O})_{\mathrm{i}}(\%)$ & 70,87 & 83,11 & 85,16 & 42,03 \\
\hline$(\mathrm{M} \mathrm{O})_{\mathrm{f}}(\%)$ & 30,31 & 32,12 & 40,20 & 32,28 \\
\hline Relação $(\mathrm{C} / \mathrm{N})_{\mathrm{i}}$ & 85,6 & 513,0 & 87,6 & 179,6 \\
\hline Relação $(\mathrm{C} / \mathrm{N})_{\mathrm{f}}$ & 16,49 & 19,50 & 12,26 & 22,41 \\
\hline Potássio $_{\mathrm{i}}(\%)$ & 0,30 & 0,06 & 0,58 & 0,06 \\
\hline Potássio $_{\mathrm{f}}(\%)$ & 1,32 & 1,49 & 1,71 & 1,26 \\
\hline Fósforo $_{i}(\%)$ & 0,083 & 0,005 & 0,053 & 0,005 \\
\hline Fósforo $_{\mathrm{f}}(\%)$ & 0,82 & 0,88 & 0,91 & 0,67 \\
\hline Massa Específica $(\%)$ & 125 & 111 & 271 & 338 \\
\hline Massa Específica $(\%)$ & 623 & 507 & 484 & 424 \\
\hline
\end{tabular}

" $\mathrm{i}=$ início; $\mathrm{f}=$ final; $\mathrm{MO}=$ matéria orgânica

a maturação/estabilização desses leitos. Estudos de compostagem (leiras estáticas aeradas e reviradas) do material retirado das camas foram implementados, os quais se mostraram eficientes para a sua humificação; finalmente, os resultados dos trabalhos de observação dos animais em criação nas camas, mostraram a sua boa adaptação, observando-se que os mesmos se apresentaram calmos e pouco estressados.

\section{Tratamento de dejetos de suínos em lagoas}

O sistema de tratamento dos dejetos de suínos, constituído de lagoas naturais, compreende as etapas apresentadas na Figura 2. Na Tabela 2 globalizam-se os resultados operacionais, obtidos através dos estudos realizados por Medri (1997). Para este trabalho foi construído e monitorado um sistema de lagoas em escala real, cujos resultados indicam a potencialidade de aplicação desta tecnologia para o tratamento dos dejetos de suínos para o Sul do Brasil e, ao mesmo tempo, apontam para a necessidade de otimização dos parâmetros de projeto, de forma a reduzir as dimensões das lagoas e garantir o desempenho hidrodinâmico nas unidades.

No estudo foi mostrada a viabilidade econômica do processo com lagoas, em comparação com propostas que necessitam de equipamentos para o tratamento biológico.

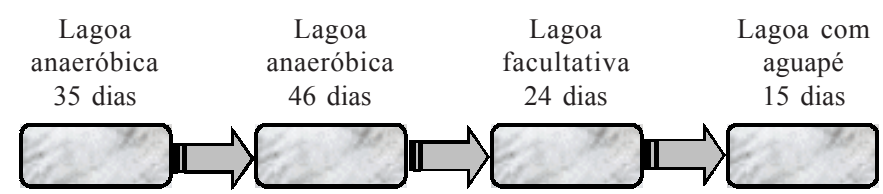

Figura 2. Sistema de lagoas avaliado no CNPSA/EMBRAPA

(Medri, 1997)
Apesar da elevada eficiência do sistema, este necessita de um pós-tratamento, para atender aos padrões de emissões de efluentes líquidos. Os resultados indicaram as lagoas funcionando como reatores de mistura completa e que a degradação da matéria orgânica segue reações com cinética de primeira ordem.

\section{Tratamento de dejetos de suínos com reator anaeróbio de fluxo ascendente com manta de lodo (Reator UASB)}

O estudo conduzido por Medri (1997) mostrou que as lagoas anaeróbias para tratar dejetos de suínos possuem muitas vantagens, porém necessitam de elevado tempo de retenção hidráulico, conforme pode ser observado na Figura 2. Desta forma, avaliou-se a possibilidade de aplicação dos reatores anaeróbios com manta de lodo de fluxo ascendente (reator UASB) para tratar dejetos de suínos, considerando que são aplicados para diversos resíduos com volumes menores, em comparação às lagoas anaeróbias.

Os reatores UASB possuem facilidades operacionais, hidrodinâmica mais eficiente que as lagoas e boa adaptação às condições climáticas do Brasil, para diversos efluentes líquidos. A concepção desta unidade foi apresentada por Lettinga (1980). Portanto, diante da possibilidade de utilização deste processo para o tratamento de dejetos de suínos, uma pesquisa foi realizada através de um sistema piloto, com volume de $136 \mathrm{~L}$, trabalhando com alimentação contínua e temperatura entre 25 a $30^{\circ} \mathrm{C}$.

Os dejetos antes de serem tratados pelo processo biológico foram micro-peneirados ( $\#=0,1 \mathrm{~mm})$, equalizados durante 15 dias e, posteriormente, encaminhados para alimentação do reator UASB, através de bomba peristáltica. O reator foi operado com tempo de detenção hidráulico de 72 e 36 h, obtendo-se cargas orgânicas respectivas de 0,8 e

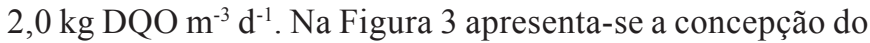

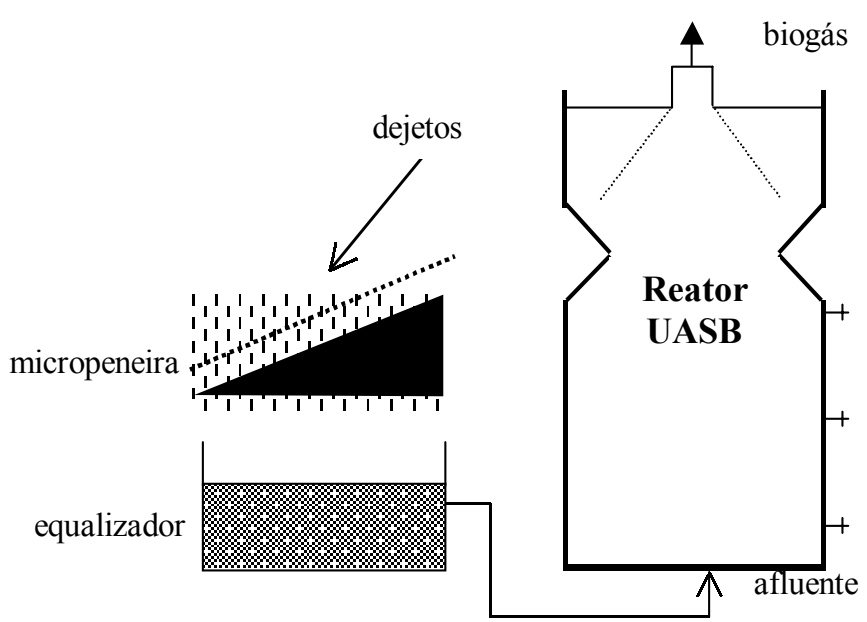

Figura 3. Concepção do sistema-piloto com reator UASB, para tratar dejetos de suínos

Tabela 2. Eficiência do sistema de lagoas naturais para tratar dejetos de suínos (Medri, 1997)

\begin{tabular}{|c|c|c|c|c|c|c|c|}
\hline \multirow{2}{*}{ Avaliação Global } & ST & SVT & DQO & $\mathrm{DBO}_{5}$ & NTK & $\mathrm{P}$ & \multirow{2}{*}{$\begin{array}{c}\text { CF } \\
\left(\mathrm{NMP} 100 \mathrm{~mL}^{-1}\right)\end{array}$} \\
\hline & \multicolumn{6}{|c|}{$\left(\mathrm{g} \mathrm{L}^{-1}\right)$} & \\
\hline Entrada do sistema & 16,7 & 10,2 & 21,8 & 10,4 & 2,2 & 1,6 & $6.10^{9}$ \\
\hline Saída do sistema & 1,3 & 0,6 & 0,4 & 0,2 & 0,18 & 0,1 & $2,7 \cdot 10^{3}$ \\
\hline Redução (\%) & 92 & 94 & 98 & 98 & 92 & 94 & \\
\hline
\end{tabular}

ST - Sólidos totais; SVT - Sais voláteis totais; DQO - Demanda química de oxigênio; DBO $_{5}$ - Demanda biológica de oxigênio; NTK - Nitrogênio total Kjedual; P - Fósforo; CF - Coliformes fecais 
Tabela 3. Resultados do armazenamento dos dejetos em esterqueira e bioesterqueira (Gosmann, 1997)

\begin{tabular}{|c|c|c|c|c|c|c|c|c|c|c|}
\hline \multirow{3}{*}{$\begin{array}{l}\text { Parâmetros } \\
\left(\mathrm{g} \mathrm{kg}^{-1}\right)\end{array}$} & \multicolumn{5}{|c|}{$1^{\mathrm{a}}$ Etapa (julho a novembro) } & \multicolumn{5}{|c|}{$2^{\mathrm{a}}$ Etapa (novembro a março) } \\
\hline & \multirow{2}{*}{$\begin{array}{c}\begin{array}{c}\text { Dejetos } \\
\text { Brutos }\end{array} \\
\text { Início }\end{array}$} & \multicolumn{2}{|c|}{ Bioesterqueira } & \multicolumn{2}{|c|}{ Esterqueira } & \multirow{2}{*}{$\begin{array}{c}\begin{array}{c}\text { Dejetos } \\
\text { Brutos }\end{array} \\
\text { Início }\end{array}$} & \multicolumn{2}{|c|}{ Bioesterqueira } & \multicolumn{2}{|c|}{ Esterqueira } \\
\hline & & $120 \mathrm{DA}^{*}$ & $\begin{array}{l}\text { Red. } \\
(\%)\end{array}$ & $120 \mathrm{DA}^{*}$ & $\begin{array}{l}\text { Red. } \\
(\%)\end{array}$ & & $120 \mathrm{DA}^{*}$ & $\begin{array}{c}\text { Red. } \\
(\%)\end{array}$ & $120 \mathrm{DA}^{*}$ & $\begin{array}{l}\text { Red. } \\
(\%)\end{array}$ \\
\hline ST & 32,9 & 29,2 & 9 & 22,9 & 30 & 32,6 & 15,6 & 52 & 14,5 & 56 \\
\hline SVT & 25,4 & 21,5 & 15,5 & 14,6 & 43 & 25,4 & 9,0 & 66 & 8,0 & 70 \\
\hline DQO & 4,1 & 36,3 & 16 & 23,3 & 46 & 44,7 & 13,4 & 70 & 13,5 & 70 \\
\hline NTK & 3,2 & 3,3 & - & 2,9 & - & 3,0 & 2,3 & - & 2,4 & - \\
\hline $\mathrm{NH}_{4}^{+}$ & 1,9 & 2,5 & - & 2,3 & - & 1,7 & 1,9 & - & 1,8 & - \\
\hline $\mathrm{P}_{2} \mathrm{O}_{5}$ & 2,2 & 2,5 & - & 2,4 & - & 2,2 & 1,1 & - & 1,6 & - \\
\hline $\mathrm{K}_{2} \mathrm{O}$ & 2,0 & 2,3 & - & 2,5 & - & 1,5 & 1,8 & - & 1,7 & - \\
\hline
\end{tabular}

${ }^{*} \mathrm{DA}$ - Dias após

Red . - redução

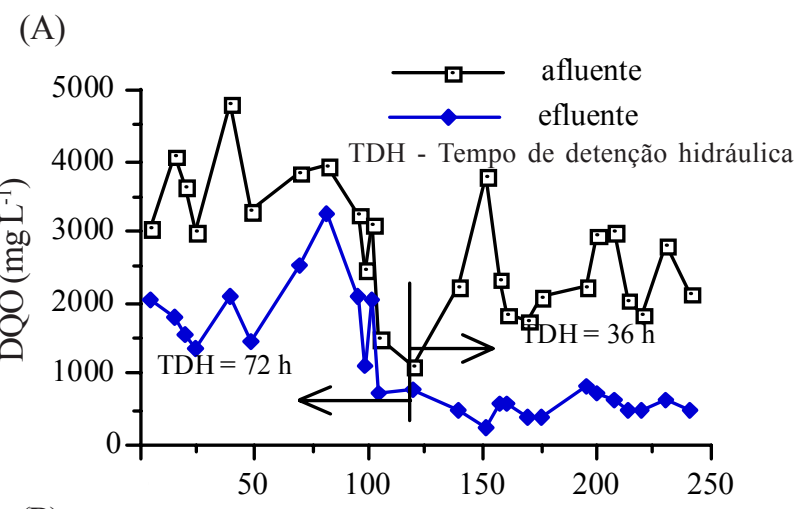

(B)

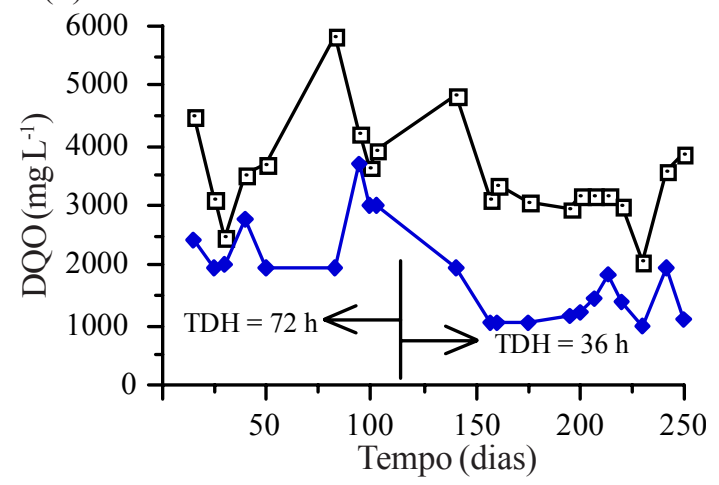

Figura 4. Comportamento do reator UASB para os parâmetros (A) ST e (B) DQO (Carmo, 1998)

reator e, na Figura 4, a eficiência obtida através dos estudos de Carmo (1998). Os resultados mostram a evolução em função do tempo para a DQO e ST, cujos valores de redução desses parâmetros, após 150 dias de operação, estão na ordem de 85 e $60 \%$, indicando que o reator UASB pode apresentar-se como alternativa às lagoas anaeróbias, no sistema de Medri (1997).

\section{Sistemas de armazenamento de dejetos de suínos}

Os dois processos mais empregados para o armazenamento dos dejetos de suínos em Santa Catarina, são os modelos conhecidos como esterqueira e bioesterqueira, conforme ilustrado na Figura 5. Esses sistemas possuem a função principal de armazenar os dejetos antes de aplicá-los ao solo, porém esses reatores biológicos proporcionam redução da fração
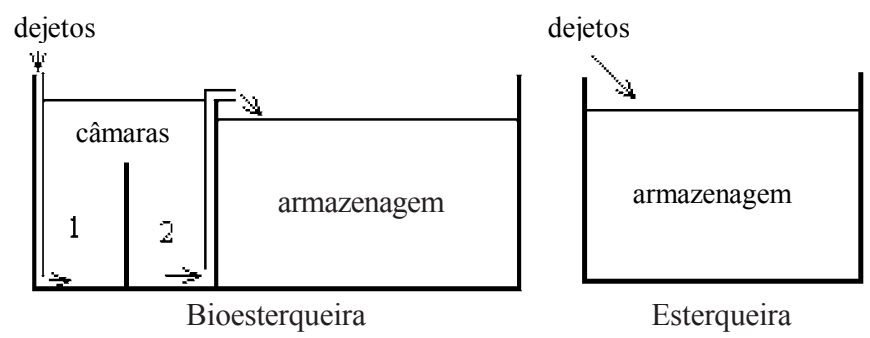

Figura 5. Modelo de bioesterqueira e esterqueira

orgânica associada à sua liquefação, preservando o potencial de fertilização deste produto.

Esses sistemas devem ser construídos para armazenar os dejetos durante o período de 120 a 180 dias, em função das características do solo e do tipo de cultura a ser desenvolvida. Desta forma, fica integrada a prática agrícola, aproveitando-se o potencial fertilizante dos dejetos com a capacidade-suporte do ecossistema solo. As experiências com protótipos dessas instalações mostraram que as duas tecnologias demonstraram comportamento semelhante, apresentados resumidamente na Tabela 3 (Gosmann, 1997); no entanto, a esterqueira possui custo de implantação e de operação inferior a bioesterqueira.

\section{CONCLUSÃO}

O problema da gestão dos dejetos de suínos é complexo e não existe, a priori, uma única solução, mas diversas possibilidades apresentam pontos positivos e negativos. As pesquisas desenvolvidas até o momento oferecem resultados motivadores que orientam para a otimização, em campo, desses procedimentos, e de novas alternativas que integrem a produtividade de suínos com a preservação ambiental. Muita atenção deve ser direcionada para ações de prevenção a médio e longo prazo.

\section{LITERATURA CITADA}

Carmo Jr., G.N.R. Aplicabilidade do reator anaeróbio de fluxo ascendente com manta de lodo (UASB) para o tratamento de resíduos líquidos de suinocultura. Florianópolis: Universidade Federal de Santa Catarina, 1998. 79 p. Dissertação Mestrado 
Gosmann, H.A. Estudos comparativos com bioesterqueira e esterqueira para armazenagem e valorização dos dejetos de suínos. Florianópolis: Universidade Federal de Santa Catarina, 1997. 115p. Dissertação de Mestrado

Goulart, R.M. Processo de compostagem: alternativa complementar para tratamento de camas biológicas de dejetos de suínos. Florianópolis: Universidade Federal de Santa Catarina, 1997. 127 p. Dissertação de Mestrado

Huysman, C.N. Experiences on 18 farms with pig on deep litter. In: Voermans, J.A.M. (ed.). Workshop Deep Litter Systems for Pig Farming. Proceedings... Rosmalen-Netherlands: Research Institute of Pig Husbandry, 1992. p.1-7.
Lettinga, G. Use of upflow sludge blanket (USB) reactor concept for biological wastewater treatment especially for anaerobic treatment. Biotechnological Bioengineering, v.22, p.699-734, 1980.

Medri, V. Modelagem e otimização de sistemas de lagoas de estabilização para tratamento de dejetos de suínos. Florianópolis: Universidade Federal de Santa Catarina, 1997. 206 p. Tese de Doutorado

Simoni, A. Allevamento di suini stalletti con lettiera permanente prime prove sperimentali. Revista di suinocultura, v.4, p.125-131, 1992.

Tumelero, I.L. Avaliação de materiais para sistema de criação de suínos sobre camas. Florianópolis: Universidade Federal de Santa Catarina, 1998. 89 p. Dissertação Mestrado 